\title{
Incomplete Abortion
}

National Cancer Institute

\section{Source}

National Cancer Institute. Incomplete Abortion. NCI Thesaurus. Code C50608.

The uterus is not entirely emptied of products of conception following spontaneous, medical or operative pregnancy termination. 